Valenciennes ED was incorrect, and that pharmacists' involvement could improve information gathering about home medicines.

During the study, pharmacists did not find any discrepancies with home meds or any drug-related problems (DRPs) in $38.2 \%$ of the patients. Pharmacists did not add value for these patients.

Separately, the Centre Hospitalier de Valenciennes pharmacy has automated the drug dispensing process. As a result, pharmacy technicians have expressed their reluctance to only work with a machine, fearing they might lose part of their skills in medicines management.

Before this problem arose, it has been proposed that technicians take part in medicines reconciliation in the ED.

Purpose To assess which tasks could be conducted by a pharmacy technician in medicines reconciliation.

Materials and Methods Technicians were present at the ED with a pharmacist. Technicians conducted standardised procedures, such as contacting the community pharmacy or assessing patients' compliance according to scores, and reported the conclusions to the pharmacist.

Results Pharmacy technicians had a strong incentive to get involved, as it refreshed their knowledge of medicines management. Moreover, it helped pharmacist to reconcile more patients in the ED, and to focus on patients with DRPs.

However, pharmacy technicians need to be trained on how to detect DRPs, such as therapeutic escalation, and on how to conduct a patient interview.

Conclusions Involving pharmacy technicians in medicines reconciliation may help the pharmacist in the ED, and allow the technicians to keep up their medicines management skills.

No conflict of interest.

\section{CPC-078 IPILIMUMAB FOR ADVANCED MELANOMA: DRUG USE REVIEW}

doi:10.1136/ejhpharm-2013-000276.535

'AR Rubio Salvador, '2J Medina Martínez, 'JM Martínez Sesmero, 'P Moya Gómez, ${ }^{2}$ MA Cruz Mora, ${ }^{2} \mathrm{JI}$ Chacón López-Muñiz, ' $J J$ Cía Lecumberri. 'Hospital Virgen de la Salud, Pharmacy, Toledo, Spain; ${ }^{2}$ Hospital Virgen de la Salud, Oncology, Toledo, Spain

Background Ipilimumab is a recombinant, fully human monoclonal antibody (IgG1) which blocks the inhibitory effects of cytotoxic T-lymphocyte antigen 4 (CTLA4), a negative regulator of T-cell activation. It has been approved for the treatment of unresectable or metastatic melanoma in patients who have failed or do not tolerate other systemic treatment for advanced disease.

Purpose To review the effectiveness and safety profile of ipilimumab in the treatment of adult patients with advanced melanoma. Materials and Methods Medical record review and retrospective analysis (January 2011 to September 2012) of prescriptions recorded in the Integral Oncology Patient Information System (ONCOBASS) in a teaching general hospital. Previous drug use, dose, line of chemotherapy, number of cycles administered, objective response rate and toxicity were analysed.

Results A total of 5 patients with metastatic melanoma were prescribed ipilimumab (2 male, 3 female), median age 45 (36-60). The 4 cycles of treatment planned were completed by 3 patients, 1 continues in active treatment at the moment of finishing this study and the other one has been lost to follow-up due to change of hospital.

In the group of four patients who received treatment, 2 were prescribed ipilimumab as a second line after failure of a temozolomide-based regimen, and 2 were prescribed ipilimumab as third line after two regimens based on immunotherapy, temozolomide or vemurafenib.

After completing the 4 cycles planned, 1 patient maintained complete response (16 months) and 1 patient showed stable disease (maintained for 5 months), and the other one is in evaluation.

No patients suffered grade 3-4 toxicity and the treatment was well tolerated.
Conclusions Ipilimumab has shown effectiveness and safety in the treatment of unresectable or metastatic melanoma in patients who have failed or do not tolerate other systemic treatment for advanced disease in our patients, although data from more patients and longer-term studies are required.

No conflict of interest.

\section{CPC-079 MANAGEMENT OF MYELODISPLASTIC SYNDROMES AND LYMPHOMAS: THE EXAMPLE OF LENALIDOMIDE}

doi:10.1136/ejhpharm-2013-000276.536

${ }^{1} \mathrm{M}$ Scaldaferri, ${ }^{1} \mathrm{E}$ Sciorsci, ${ }^{1} \mathrm{~F}$ Re, ${ }^{2} \mathrm{C}$ Calvo, ${ }^{3} \mathrm{M}$ Chiumente, ${ }^{3} \mathrm{D}$ Barilà, ${ }^{3} \mathrm{~A}$ Chiesa, ${ }^{3}$ M Ferroni, 'S Stecca, 'F Cattel. 'A. O.U. San Giovanni Battista, Pharmacy, Turin, Italy; ${ }^{2}$ University of Turin, Faculty of Pharmacy, Turin, Italy; ${ }^{3}$ University of Turin, School of Hospital Pharmacy, Turin, Italy

Background At our centre, haematologists and department pharmacists constantly monitor outcomes and safety of treatment with lenalidomide.

Purpose To describe clinical outcomes and safety of lenalidomide in our lymphoma and myelodysplastic syndrome patients.

Materials and Methods Onco-AIFA Registry and medical records were checked as of 30/06/2012 for diagnosis, duration of treatment, incidence of adverse drug events (ADRs).

Results Data of 34 patients were reviewed, with the following diagnoses: Diffuse large B-cell lymphoma (DLBCL), 24 patients; $5 \mathrm{q}$-myelodysplastic syndrome (MDS5q-), 11 patients and mantle cell lymphoma (MCL), one patient.

Of patients with DLBCL, one discontinued treatment because of serious ADRs, two because of death and 4 for disease progression after an average of 4.4 treatment cycles, corresponding to 7 months (range: $2-18$ ).

Of patients with MDS5q-, 8 stopped treatment, two of whom because of disease progression or death and two for toxicity. The median duration of treatment was 11.8 cycles (range 1-29).

Seventeen DLBCL patients and 3 MDS5q- patients are still on therapy.

34 non-serious $\mathrm{ADR}$ s relating to 14 patients and 5 serious $\mathrm{ADR}$ relating to 4 patients were reported, two of which were cases of development of solid neoplasia. Non-serious ADRs were mostly cases of haematological toxicity, alterations of the skin and of nervous system and infections.

Conclusions Lenalidomide seems to control the disease in patients with MDS5q- for long periods, while the Time to Progression in patients with DLBCL appears shorter.

The treatment-related toxicity appears in most cases acceptable.

Despite the limited number of data, our analysis highlights the need for close monitoring of the patients both during treatment and on follow-up, as evidenced by the two cases of onset of neoplasia.

The progressive collection of data is providing the haematologists and pharmacists the information to design a model for optimised appropriate treatment with lenalidomide.

No conflict of interest.

\section{CPC-080 MANAGEMENT OF POSTOPERATIVE PAIN AT MOHAMMED V MILITARY TEACHING HOSPITAL, RABAT, MOROCCO}

doi:10.1136/ejhpharm-2013-000276.537

'W Enneffah, "I Zakariya, 'MA El Wartiti, ${ }^{2} \|$ Fabrice, ${ }^{3} \mathrm{~N}$ Cherkaoui, ${ }^{1} \mathrm{~A}$ Bennana. ${ }^{1}$ Mohammed V Military Teaching Hospital - Faculty of Medicine and Pharmacy, Clinical Pharmacy - Therapeutic Chemistry, Rabat, Morocco; ${ }^{2}$ Faculty of Medicine and Pharmacy - Mohammed V University, Souissi, Rabat, Morocco; ' 3 ohammed V Military Teaching Hospital - Faculty of Medicine and Pharmacy, Pharmacy - Galenic Pharmacy, Rabat, Morocco 